\title{
DFT, molecular docking and molecular dynamics simulation studies on natural chromone derivatives from Cassia nomame for their possible antiviral activity against Coronavirus, SARS-CoV-2
}

\author{
Taner ERDOGAN ${ }^{1, *}$ \\ ${ }^{1}$ Kocaeli University, Kocaeli Vocational School, Department of Chemistry and Chemical Processing Technologies, \\ Kocaeli / TURKEY
}

\begin{abstract}
In this study, two naturally occurred chromone derivatives obtained from Cassia nomame which are recently entered the literature, have been investigated computationally for their potential antiviral activity against SARS-CoV-2. In the first part of the study, DFT calculations were performed on the investigated compounds. In this part, geometry optimizations, frequency analyses, molecular electrostatic potential map calculations, frontier molecular orbital calculations and NMR spectral studies have been performed. In the second part of the study, molecular docking calculations were performed. SARS-CoV-2 main protease (SARSCoV-2 $\mathrm{M}^{\text {pro }}$ ) was selected as receptor for molecular docking calculations. In the third part of the study, molecular dynamics simulation studies were performed on the top scoring SARSCoV-2 $\mathrm{M}^{\text {pro }}$ - ligand complexes. In this part, binding free energy calculations were also performed on the SARS-CoV-2 $\mathrm{M}^{\text {pro }}$ - ligand complexes with the use of molecular mechanics with Poisson-Boltzmann surface area (MM-PBSA) method. Results showed that, two naturally occurred chromone derivatives, 5-(isobutyryl)-2-(2-oxopropyl)-7-methoxy-4Hchromen-4-one and 5-(isobutyryl)-2-(2-oxopropyl)-6-methoxy-4H-chromen-4-one, showed quite high binding affinity to SARS-CoV-2 $\mathrm{M}^{\text {pro }}$ and remained stable during the molecular dynamics simulations. Additionally, in the last part of the study, drug-likeness analyses were performed on the investigated compounds with the use of Lipinski's rule of five and no violation was observed.
\end{abstract}

\section{Article info}

History:

Received: 16.05.2020

Accepted: 14.07.2021

Keywords:

COVID-19,

Chromone derivatives,

Cassia nomame,

Molecular docking,

Molecular dynamics

simulation.

\section{Introduction}

Since its first appearance in China in December 2019, SARS-CoV-2 has been causing human pulmonary infections and despite all the measures taken, it continues to spread all over the world very quickly. Currently, vaccination is the only effective way to treat COVID-19, and unfortunately, a specific drug treatment has not been developed yet, and it is a critical issue to propound specific drug treatment options today and in near future.

Drug treatment investigations for SARS-CoV-2 infections can be divided into two groups: (1) To use the drugs currently used in SARS and MERS treatments due to the similarity between the target proteins and (2) to develop new specific drugs. Currently, intensive studies are being conducted on whether drugs used in the treatment of SARS and MERS can be used against SARS-CoV-2 such as favipiravir, ribavirin, remdesivir, galidesivir, disulfiram, lopinavir, ritonavir etc. and other synthesized or isolated compounds found in databases are being investigated against SARS-CoV-2. [1-8] Since it has an important role in viral replication and transcription, SARS-CoV-2 $\mathrm{M}^{\text {pro }}$ is a commonly selected target in drug repurposing and new drug development studies for the treatment of COVID-19. $[9,10]$

In this study, two novel natural products which were isolated by Liao et al. [11] from Cassia nomame have been investigated computationally for their possible antiviral activity against SARS-CoV-2 via performing molecular docking calculations and molecular dynamics simulations. The structures of these natural chromone derivatives are given in Figure 1. 
In the first part of the study, density functional theory calculations were performed on the investigated compounds.

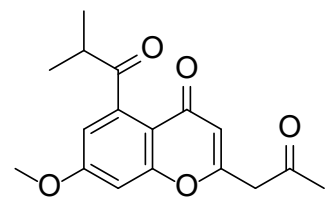

5-(isobutyryl)-2-(2-oxopropyl)-7-methoxy-4H-chromen-4-one Comp. 1

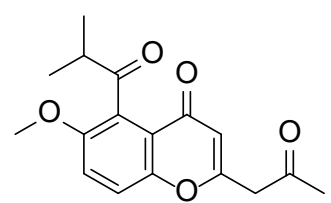

5-(isobutyryl)-2-(2-oxopropyl)-6-methoxy-4H-chromen-4-one Comp.2

Figure 1. Chemical structures of Comp. 1 and 2.

Geometry optimizations, frequency analyses, MEP (molecular electrostatic potential) map calculations, frontier molecular orbital (FMO) calculations and NMR spectral studies were carried out. In the second part, molecular docking calculations were performed, and then molecular dynamics simulations were carried out on the top-scoring enzyme - ligand complexes, and binding free energies were determined. Finally, druglikeness analyses were performed on the investigated compounds.

\section{Materials and Methods}

\subsection{DFT calculations}

In this part, the title compounds have been investigated computationally and geometry optimizations, frequency analyses, MEP map calculations, FMO calculations and NMR spectral analyses were performed. In DFT calculations, Gaussian 09 Rev.D.01 [12], GaussView 5 [13], VeraChem VConf [14] and Avogadro 1.1.1 [15] software packages were used. All calculations were performed with the use of DFT B3LYP method and various basis sets including $6-31+\mathrm{G}(\mathrm{d}), \quad 6-31+\mathrm{G}(\mathrm{d}, \mathrm{p}), \quad 6-311+\mathrm{G}(\mathrm{d}, \mathrm{p})$ and 6$311+\mathrm{G}(2 \mathrm{~d}, \mathrm{p})$. Geometry optimizations were performed in gas phase. Prior to geometry optimizations, a conformational search was performed for each structure and frequency analyses were also performed to confirm that each optimized geometry corresponds to a global minimum.

\subsection{Molecular docking calculations and molecular dynamics simulations}

Geometry optimized structures of Comp. 1 and 2 were used for molecular docking calculations. In molecular docking calculations AutoDock Tools [16] and
AutoDock Vina were used and Discovery Studio Visualizer [17] was used for the representation of the docking results. 3D structure of SARS-CoV-2 $\mathrm{M}^{\text {pro }}$ was obtained from RCSB Protein Data Bank $[18,19]$ (PDB ID:5R80). Prior to molecular docking, water molecules and the bound ligands in the structure of the enzyme were removed, hydrogen atoms and Gasteiger charges were added, and docking calculations were performed with the use of Lamarckian genetic algorithm. After performing molecular docking calculations, top-scoring ligand-enzyme complexes were subjected to $30 \mathrm{~ns}$ molecular dynamics simulations. In molecular dynamics simulations, GROMACS [20] program package, AMBER [21] force field and TIP3P water model were used. Acpype Server [22] was used in the preparation of ligand topologies. After energy minimizations, 200 ps NVT and NPT ensemble equilibrations, molecular dynamics simulations were performed for $30 \mathrm{~ns}$ at 1 bar and 300 $\mathrm{K}$ reference pressure and temperature After performing molecular dynamics simulations, binding free energies were calculated with the use of MM-PBSA method for the last $20 \mathrm{~ns}$ of the MD simulations. In MM-PBSA calculations, g_mmpbsa tool $[23,24]$ was used.

\subsection{Drug-likeness analyses}

Lipinski's rule of five $[25,26]$ was used to evaluate drug-likeness of the investigated compounds. Druglikeness of the investigated compounds were tested with the use of DruLiTo software package [27].

\section{Results and Discussion}

\subsection{DFT calculations}

Optimized structures of the investigated natural compounds obtained from DFT calculations with the use of 6-311+G(2d,p) basis set are given in Figure 2. A frequency analysis was performed for each structure to confirm that the optimized structures correspond to global minima and results showed that geometry optimized structures correspond to global minima.

To obtain information about the electron deficient and the electron rich regions of the compounds under investigation, MEP map calculations were performed at the same level of theory.

MEP maps of the compounds obtained with the use of $6-311+G(2 d, p)$ basis set are given in Figure 3. It was observed that negative charge was mainly localized on the carbonyl oxygens of both compounds 1 and 2, while positive charge as located generally on the alkyl hydrogens. Molecular docking and molecular dynamics simulation results showed that these negative and positive centers took part in the interactions between investigated compounds and SARS-CoV-2 M ${ }^{\text {pro }}$. 

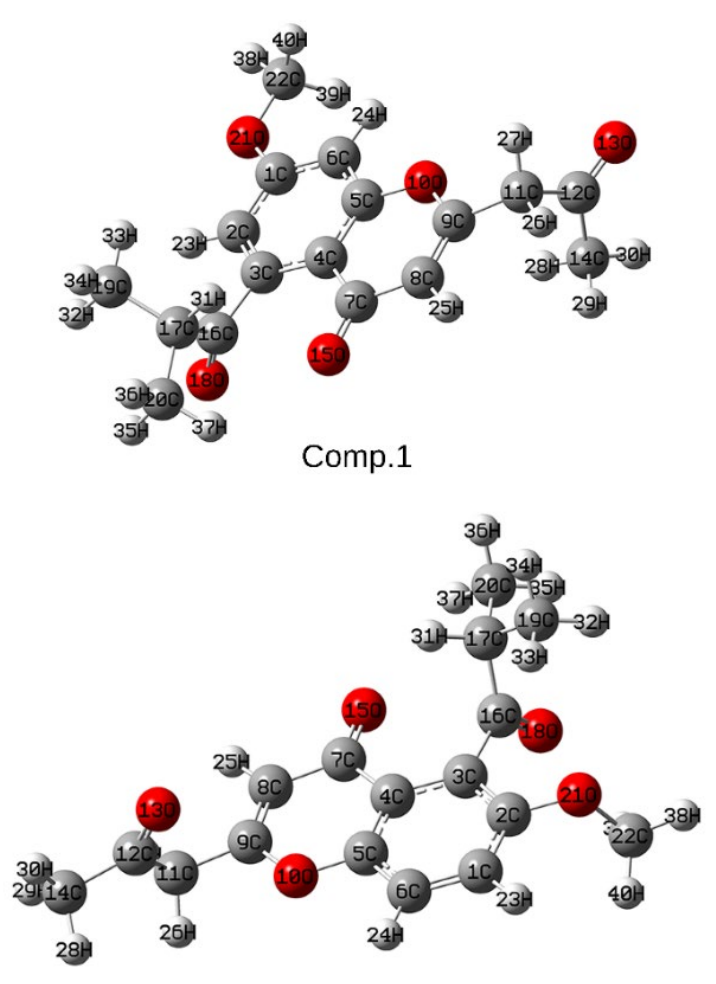

Comp.2

Figure 2. Optimized geometries of Comp. 1 and 2.

FMOs and FMO energies of the investigated compounds have also been determined and are given in Figure 4. It was observed that HOMO-LUMO gap of compound 1 is slightly greater than that of compound 2. HOMO-LUMO gap is thought to be useful parameter for examining the kinetic stability. Since compound 1 has larger HOMO-LUMO gap value, it can be said that compound 1 is more stable than compound 2. HOMO-LUMO energies and HOMO-LUMO gaps of the investigated compounds calculated with the use of $6-31+\mathrm{G}(\mathrm{d}), 6-31+\mathrm{G}(\mathrm{d}, \mathrm{p}), 6-$ $311+\mathrm{G}(\mathrm{d}, \mathrm{p})$ and $6-311+\mathrm{G}(2 \mathrm{~d}, \mathrm{p})$ basis sets are given in Table 1. All values in Table 1 are given in eV.

Nuclear magnetic shield tensors for the compounds were performed at the same level of theory with the use of GIAO method. In this part, 6-31+G(d), 6$31+\mathrm{G}(\mathrm{d}, \mathrm{p}), \quad 6-311+\mathrm{G}(\mathrm{d}, \mathrm{p})$ and $6-311+\mathrm{G}(2 \mathrm{~d}, \mathrm{p})$ basis sets were used. In NMR calculations IEFPCM solvation model was used and $\mathrm{CDCl}_{3}$ was selected as solvent.

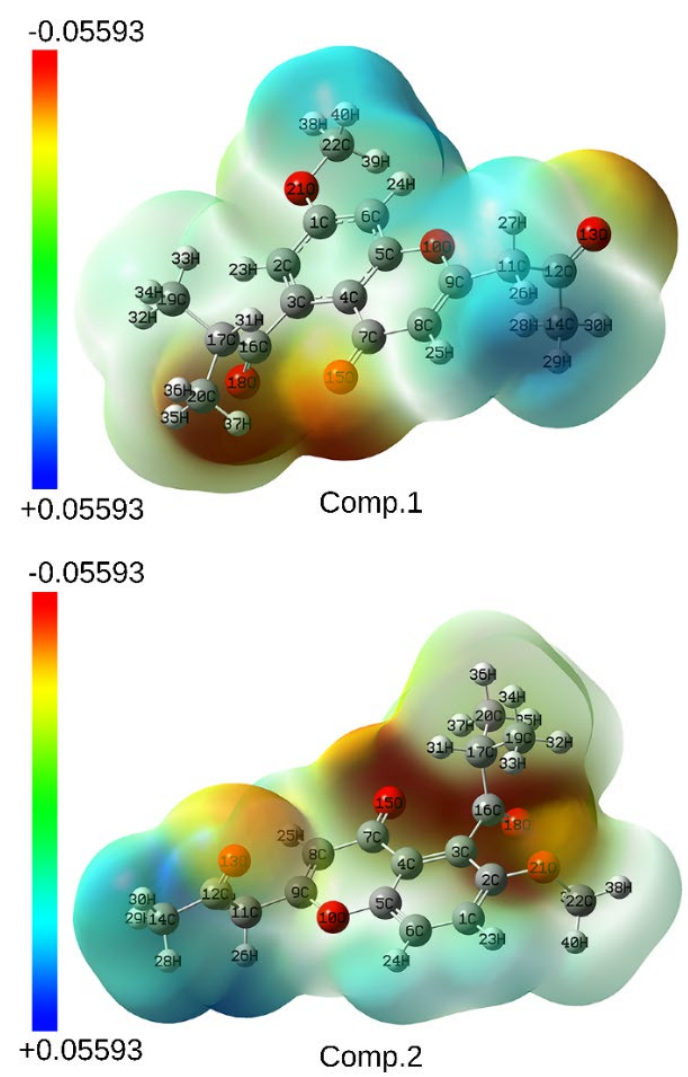

Figure 3. MEP maps of Comp. 1 and 2
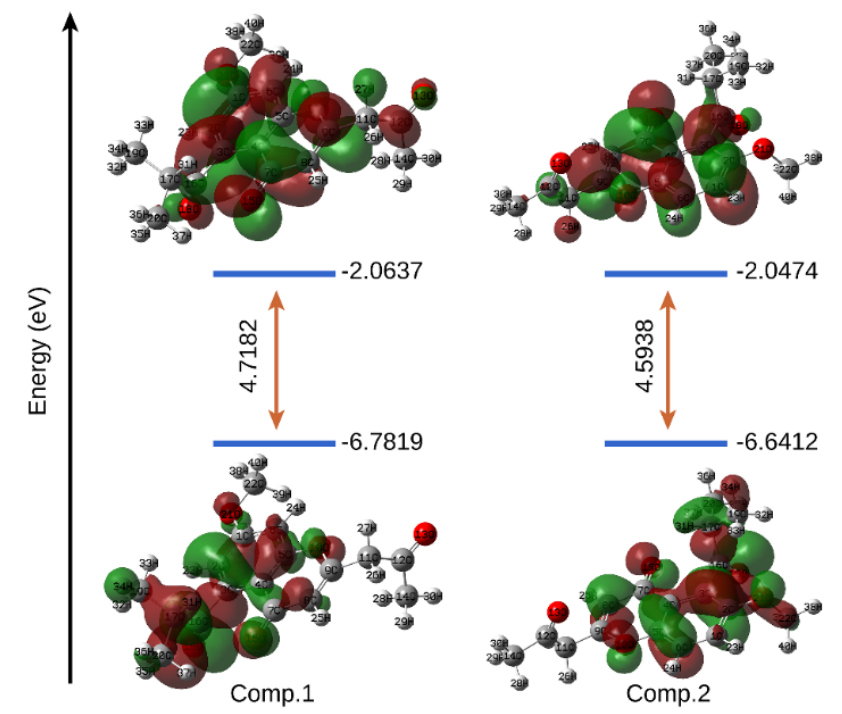

Figure 4. FMOs of Comp. 1 and 2

Calculated and experimental NMR chemical shifts reference to TMS are given in Tables 2, 3, 4 and 5. 
Table 1. Calculated FMO energies and HOMO-LUMO gaps of investigated compounds.

\begin{tabular}{lllll}
\hline Atom & $6-31+\mathrm{G}(\mathrm{d})$ & $6-31+\mathrm{G}(\mathrm{d}, \mathrm{p})$ & $6-311+\mathrm{G}(\mathrm{d}, \mathrm{p})$ & $6-311+\mathrm{G}(2 \mathrm{~d}, \mathrm{p})$ \\
\hline Comp. 1 & & & & \\
\hline LUMO & -1.7565 & -1.7228 & -2.0814 & -2.0637 \\
HOMO & -6.2488 & -6.3928 & -6.7735 & -6.7819 \\
Gap & 4.4923 & 4.6700 & 4.6921 & 4.7182 \\
\hline Comp. 2 & & & & -2.0474 \\
\hline LUMO & -1.7451 & -1.6885 & -2.0819 & -6.6412 \\
HOMO & -6.2390 & -6.2864 & -6.6682 & 4.5938 \\
Gap & 4.4939 & 4.5979 & 4.5863 & \\
\hline
\end{tabular}

It was observed that except some certain types of satisfactory results than larger basis sets and there is no hydrogen and carbon, smaller basis sets gave more need to use larger basis sets.

Table 2. Experimental and calculated ${ }^{1} \mathrm{H}-\mathrm{NMR}$ data for Comp. 1

\begin{tabular}{llllll}
\hline Atom & Exp. $[11]$ & $6-31+\mathrm{G}(\mathrm{d})$ & $6-31+\mathrm{G}(\mathrm{d}, \mathrm{p})$ & $6-311+\mathrm{G}(\mathrm{d}, \mathrm{p})$ & $6-311+\mathrm{G}(2 \mathrm{~d}, \mathrm{p})$ \\
\hline $23-\mathrm{H}$ & 7.42 & 6.72 & 6.96 & 6.97 & 7.08 \\
$24-\mathrm{H}$ & 7.12 & 6.74 & 7.04 & 7.12 & 7.23 \\
$25-\mathrm{H}$ & 6.32 & 5.95 & 6.22 & 6.27 & 6.37 \\
$26-\mathrm{H}$ & 3.55 & 3.56 & 3.63 & 3.74 & 3.83 \\
$27-\mathrm{H}$ & 3.55 & 3.56 & 3.63 & 3.74 & 3.83 \\
$28-\mathrm{H}$ & 2.17 & 2.17 & 2.26 & 2.36 & 2.40 \\
$29-\mathrm{H}$ & 2.17 & 2.17 & 2.26 & 2.36 & 2.40 \\
$30-\mathrm{H}$ & 2.17 & 2.17 & 2.26 & 2.36 & 2.40 \\
$31-\mathrm{H}$ & 4.25 & 3.05 & 3.07 & 3.09 & 3.22 \\
$32-\mathrm{H}$ & 1.23 & 1.03 & 1.04 & 1.13 & 1.13 \\
$33-\mathrm{H}$ & 1.23 & 1.03 & 1.04 & 1.13 & 1.13 \\
$34-\mathrm{H}$ & 1.23 & 1.03 & 1.04 & 1.13 & 1.13 \\
$35-\mathrm{H}$ & 1.23 & 1.46 & 1.48 & 1.58 & 1.56 \\
$36-\mathrm{H}$ & 1.23 & 1.46 & 1.48 & 1.58 & 1.56 \\
$37-\mathrm{H}$ & 1.23 & 1.46 & 1.48 & 1.58 & 1.56 \\
$38-\mathrm{H}$ & 3.82 & 3.92 & 3.99 & 4.04 & 4.06 \\
$39-\mathrm{H}$ & 3.82 & 3.92 & 3.99 & 4.04 & 4.06 \\
$40-\mathrm{H}$ & 3.82 & 3.99 & 4.04 & \\
\hline
\end{tabular}


Table 3. Experimental and calculated ${ }^{13} \mathrm{C}-\mathrm{NMR}$ data for Comp. 1

\begin{tabular}{llllll}
\hline Atom & Exp. $[11]$ & $6-31+\mathrm{G}(\mathrm{d})$ & $6-31+\mathrm{G}(\mathrm{d}, \mathrm{p})$ & $6-311+\mathrm{G}(\mathrm{d}, \mathrm{p})$ & $6-311+\mathrm{G}(2 \mathrm{~d}, \mathrm{p})$ \\
\hline 1-C & 166.8 & 157.8 & 160.0 & 172.6 & 172.6 \\
2-C & 106.2 & 108.8 & 109.8 & 119.1 & 118.7 \\
3-C & 136.8 & 142.8 & 144.8 & 154.2 & 153.9 \\
4-C & 114.8 & 113.0 & 114.8 & 123.2 & 123.2 \\
5-C & 155.4 & 153.9 & 155.8 & 166.5 & 166.8 \\
6-C & 110.2 & 99.5 & 100.5 & 108.3 & 107.7 \\
7-C & 181.4 & 169.8 & 171.6 & 183.1 & 183.8 \\
8-C & 112.8 & 110.7 & 111.8 & 120.3 & 119.9 \\
9-C & 158.2 & 159.5 & 161.4 & 172.2 & 172.5 \\
11-C & 48.3 & 51.0 & 51.9 & 55.3 & 54.9 \\
12-C & 203.1 & 200.1 & 201.6 & 215.6 & 215.6 \\
14-C & 30.4 & 29.9 & 30.3 & 32.7 & 32.4 \\
16-C & 208.5 & 209.4 & 211.2 & 226.4 & 226.0 \\
17-C & 38.3 & 46.2 & 47.0 & 49.6 & 50.0 \\
19-C & 18.6 & 21.3 & 21.6 & 21.9 & 21.5 \\
20-C & 18.6 & 21.3 & 21.6 & 21.9 & 21.5 \\
22-C & 56.2 & 55.0 & 55.2 & 58.2 & 5.1 \\
\hline
\end{tabular}

Table 4. Experimental and calculated ${ }^{1} \mathrm{H}-\mathrm{NMR}$ data for Comp. 2

\begin{tabular}{|c|c|c|c|c|c|}
\hline Atom & Exp. [11] & $6-31+G(d)$ & $6-31+G(d, p)$ & $6-311+G(d, p)$ & $6-311+G(2 d, p)$ \\
\hline $23-\mathrm{H}$ & 6.93 & 7.49 & 7.71 & 7.74 & 7.82 \\
\hline 24-H & 7.10 & 7.50 & 7.73 & 7.79 & 7.86 \\
\hline $25-\mathrm{H}$ & 6.35 & 5.99 & 6.25 & 6.31 & 6.37 \\
\hline $26-\mathrm{H}$ & 3.56 & 3.55 & 3.63 & 3.76 & 3.82 \\
\hline $27-\mathrm{H}$ & 3.56 & 3.55 & 3.63 & 3.76 & 3.82 \\
\hline $28-\mathrm{H}$ & 2.17 & 2.18 & 2.26 & 2.38 & 2.40 \\
\hline $29-\mathrm{H}$ & 2.17 & 2.18 & 2.26 & 2.38 & 2.40 \\
\hline $30-\mathrm{H}$ & 2.17 & 2.18 & 2.26 & 2.38 & 2.40 \\
\hline $31-\mathrm{H}$ & 4.27 & 3.03 & 3.05 & 3.09 & 3.16 \\
\hline $32-\mathrm{H}$ & 1.26 & 1.30 & 1.31 & 1.42 & 1.37 \\
\hline $33-\mathrm{H}$ & 1.26 & 1.30 & 1.31 & 1.42 & 1.37 \\
\hline $34-\mathrm{H}$ & 1.26 & 1.30 & 1.31 & 1.42 & 1.37 \\
\hline $35-\mathrm{H}$ & 1.26 & 1.30 & 1.31 & 1.42 & 1.37 \\
\hline $36-\mathrm{H}$ & 1.26 & 1.30 & 1.31 & 1.42 & 1.37 \\
\hline $37-\mathrm{H}$ & 1.26 & 1.30 & 1.31 & 1.42 & 1.37 \\
\hline $38-\mathrm{H}$ & 3.84 & 3.82 & 3.91 & 3.97 & 4.02 \\
\hline 39-H & 3.84 & 3.82 & 3.91 & 3.97 & 4.02 \\
\hline 40-H & 3.84 & 3.82 & 3.91 & 3.97 & 4.02 \\
\hline
\end{tabular}


Table 5. Experimental and calculated ${ }^{13} \mathrm{C}-\mathrm{NMR}$ data for Comp. 2

\begin{tabular}{llllll}
\hline Atom & Exp. $[11]$ & $6-31+\mathrm{G}(\mathrm{d})$ & $6-31+\mathrm{G}(\mathrm{d}, \mathrm{p})$ & $6-311+\mathrm{G}(\mathrm{d}, \mathrm{p})$ & $6-311+\mathrm{G}(2 \mathrm{~d}, \mathrm{p})$ \\
\hline 1-C & 120.2 & 126.2 & 127.3 & 137.6 & 137.1 \\
2-C & 156.4 & 150.6 & 152.6 & 164.3 & 164.0 \\
3-C & 121.4 & 135.2 & 137.0 & 146.6 & 146.4 \\
4-C & 118.5 & 120.0 & 121.8 & 130.7 & 130.9 \\
5-C & 150.4 & 149.4 & 151.4 & 161.6 & 161.8 \\
6-C & 122.9 & 116.1 & 117.3 & 126.7 & 126.1 \\
7-C & 181.8 & 170.5 & 172.3 & 183.8 & 184.5 \\
8-C & 110.9 & 109.9 & 111.0 & 119.6 & 119.2 \\
9-C & 159.9 & 159.9 & 161.8 & 172.4 & 172.9 \\
11-C & 48.1 & 51.7 & 52.5 & 56.0 & 55.6 \\
12-C & 203.4 & 199.9 & 201.4 & 215.1 & 215.1 \\
14-C & 30.2 & 29.8 & 30.1 & 32.6 & 32.2 \\
16-C & 208.4 & 208.4 & 210.2 & 224 & 223.7 \\
17-C & 38.2 & 47.6 & 48.4 & 50.6 & 50.8 \\
19-C & 18.6 & 21.0 & 21.3 & 21.6 & 21.2 \\
20-C & 18.6 & 21.0 & 66.6 & 69.1 & 21.2 \\
22-C & 56.2 & & & & 69.4 \\
\hline
\end{tabular}

3.2. Molecular docking calculations and molecular dynamics simulation studies

After performing molecular docking calculations, 30 ns molecular dynamics simulations were carried out.
The structures of the SARS-CoV-2 M pro - Comp. 1 complex obtained from the trajectory for every $5 \mathrm{~ns}$ of the $30 \mathrm{~ns}$ molecular dynamics simulation are given in Figure 5. 

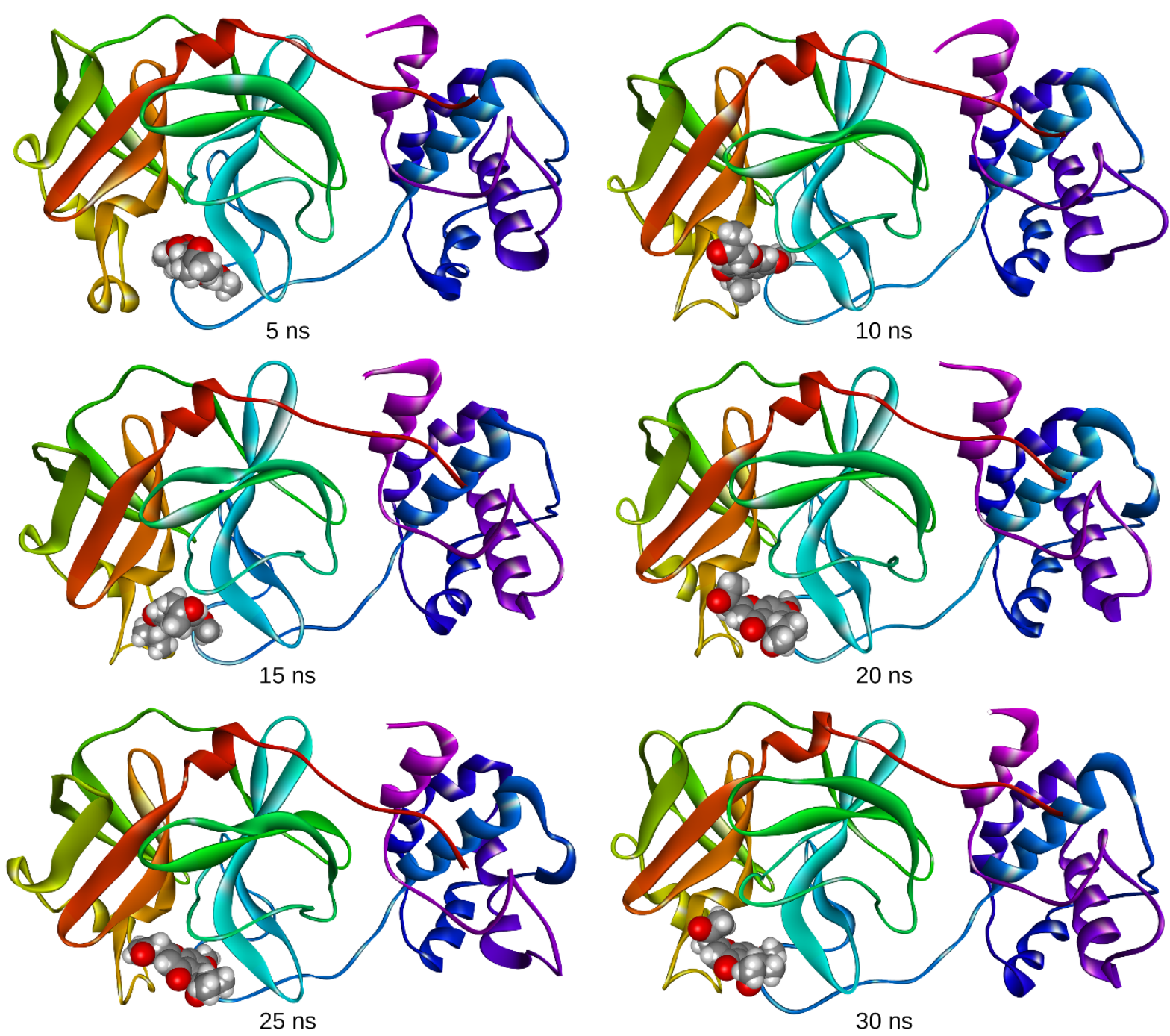

Figure 5. The structures of enzyme - Comp. 1 complex extracted from the trajectory of MD simulation.

Results showed that Comp. 1 bound to the active site of SARS-CoV-2 $\mathrm{M}^{\text {pro }}$ and held its position throughout the entire simulation. The structures of the SARSCoV-2 $\mathrm{M}^{\text {pro }}$ - Comp. 2 complex obtained from the trajectory for every $5 \mathrm{~ns}$ of the $30 \mathrm{~ns}$ molecular dynamics simulation are given in Figure 6 and it was observed that Comp. 2 also bound to the active site of the enzyme and held its position throughout the entire simulation. 

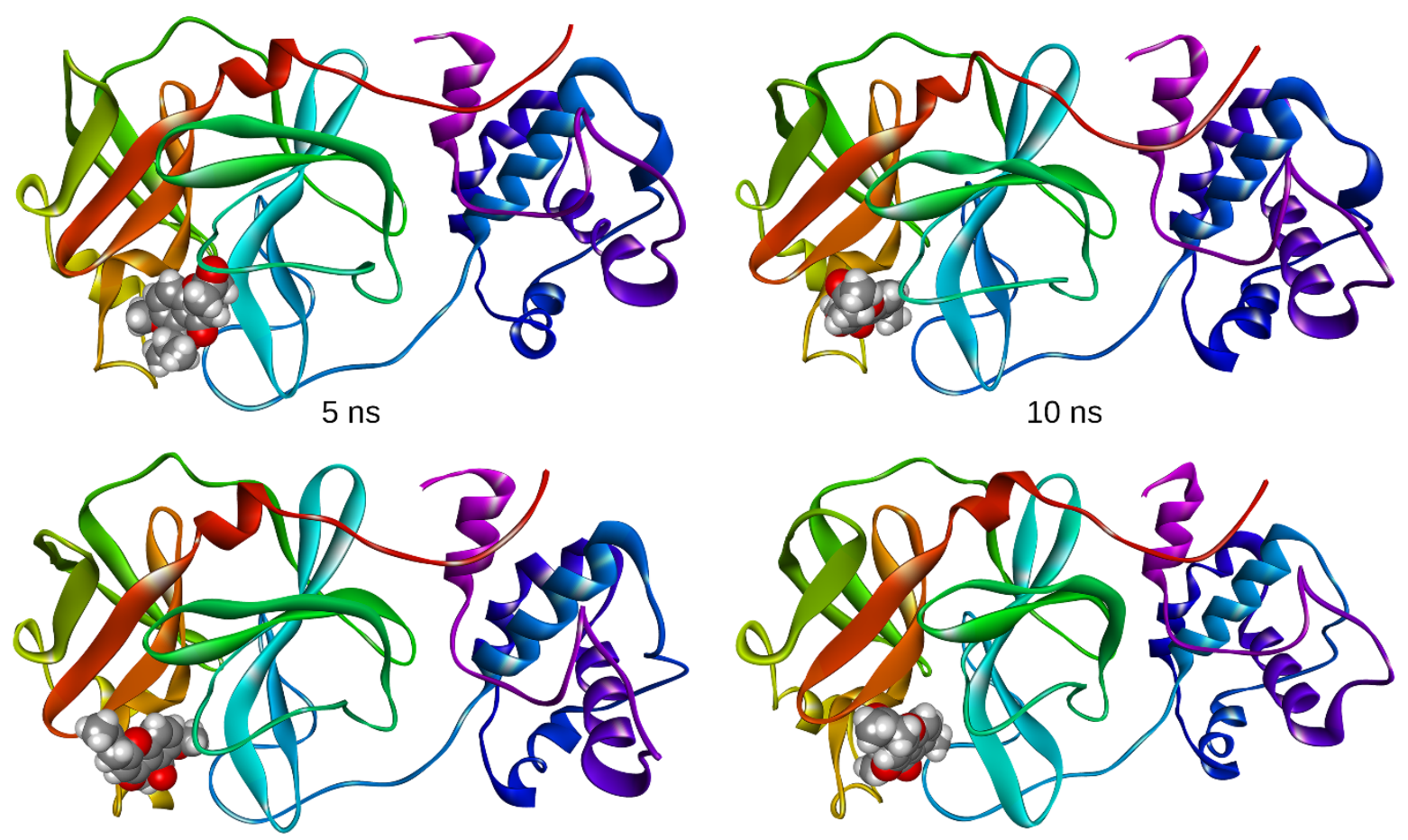

$15 \mathrm{~ns}$
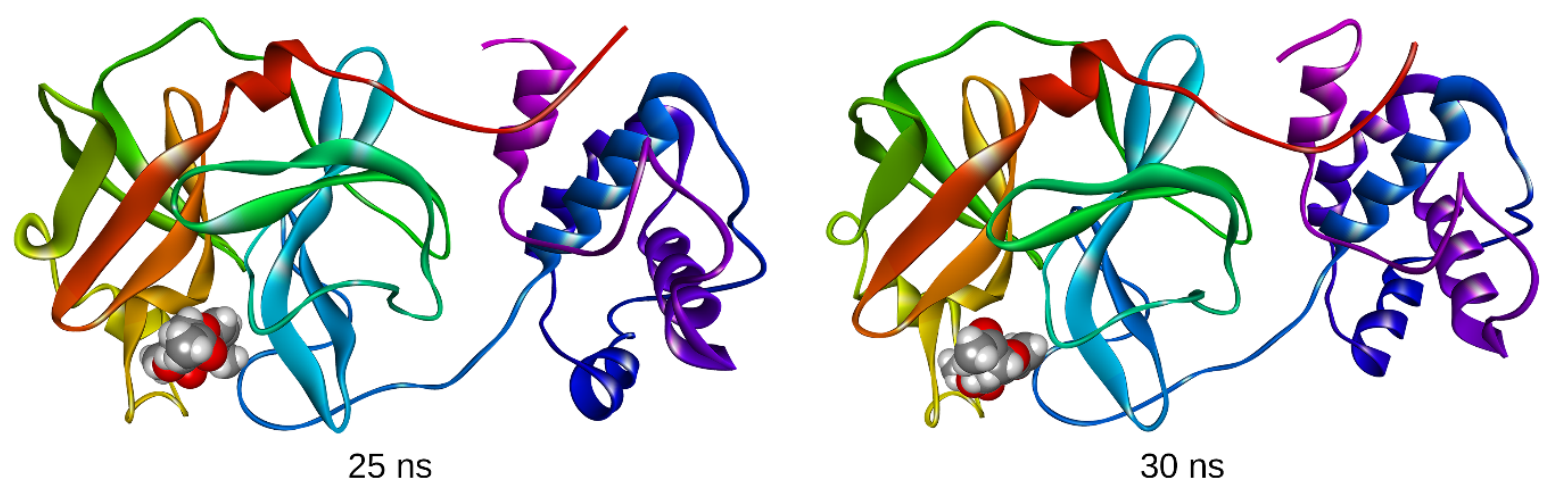

Figure 6. The structures of enzyme - Comp. 2 complex extracted from the trajectory of MD simulation.

In Figure 7, 3D and 2D interactions between investigated compounds and SARS-CoV-2 $\mathrm{M}^{\text {pro }}$ at the end of molecular dynamics simulations are illustrated. Results showed that, Comp. 1 interacted with HIS41, MET49, MET165, VAL186 and GLN189 amino acids of SARS-CoV-2 $\mathrm{M}^{\text {pro }}$. Hydrogen bonds, alkyl, $\pi$-alkyl and $\pi$-sulfur interactions took part in the stabilization of the enzyme - Comp. 1 complex. On the other hand, Comp. 2 interacted with HIS41, MET49, GLY143, CYS145 and SER46. Hydrogen bonds, alkyl and $\pi$ alkyl interactions took part in the stabilization of enzyme - Comp. 2 complex. 


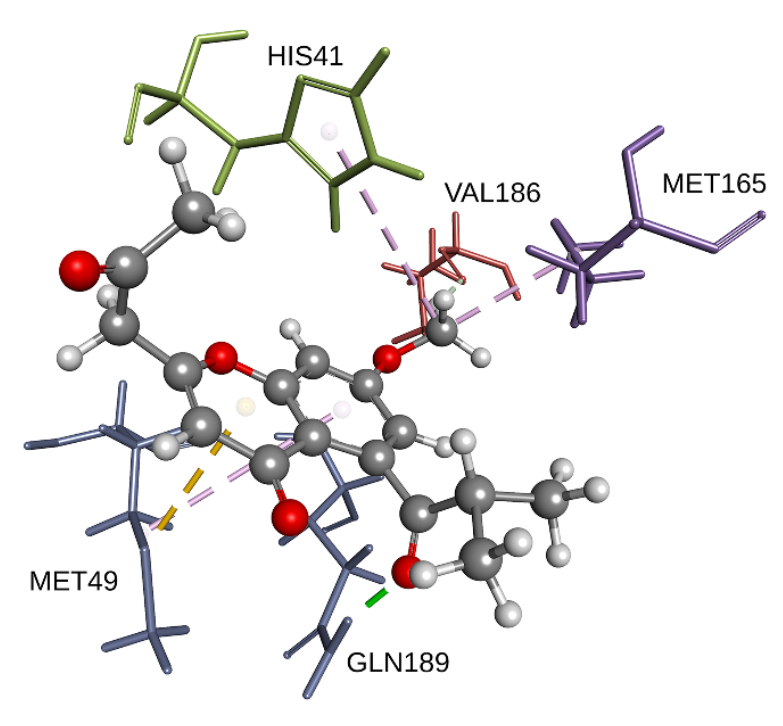

enzyme - comp.1 complex

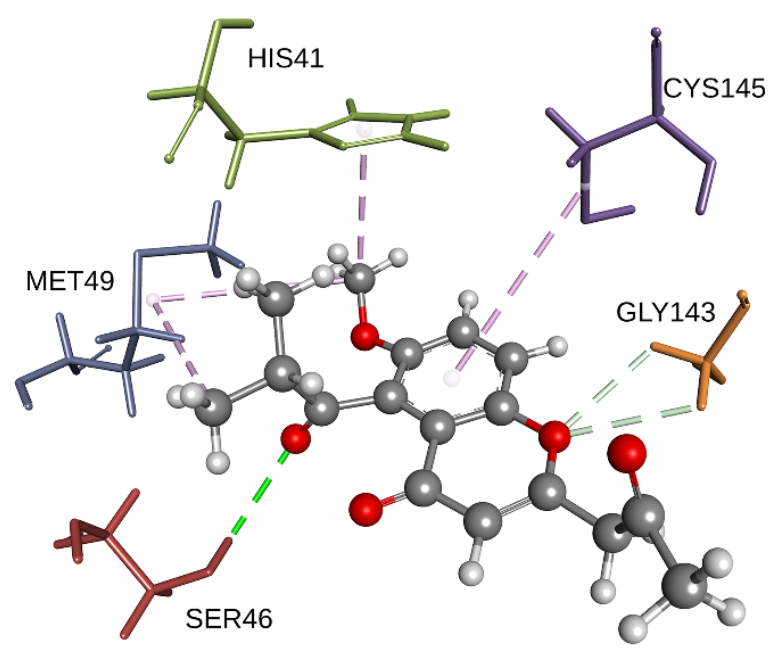

enzyme - comp. 2 complex
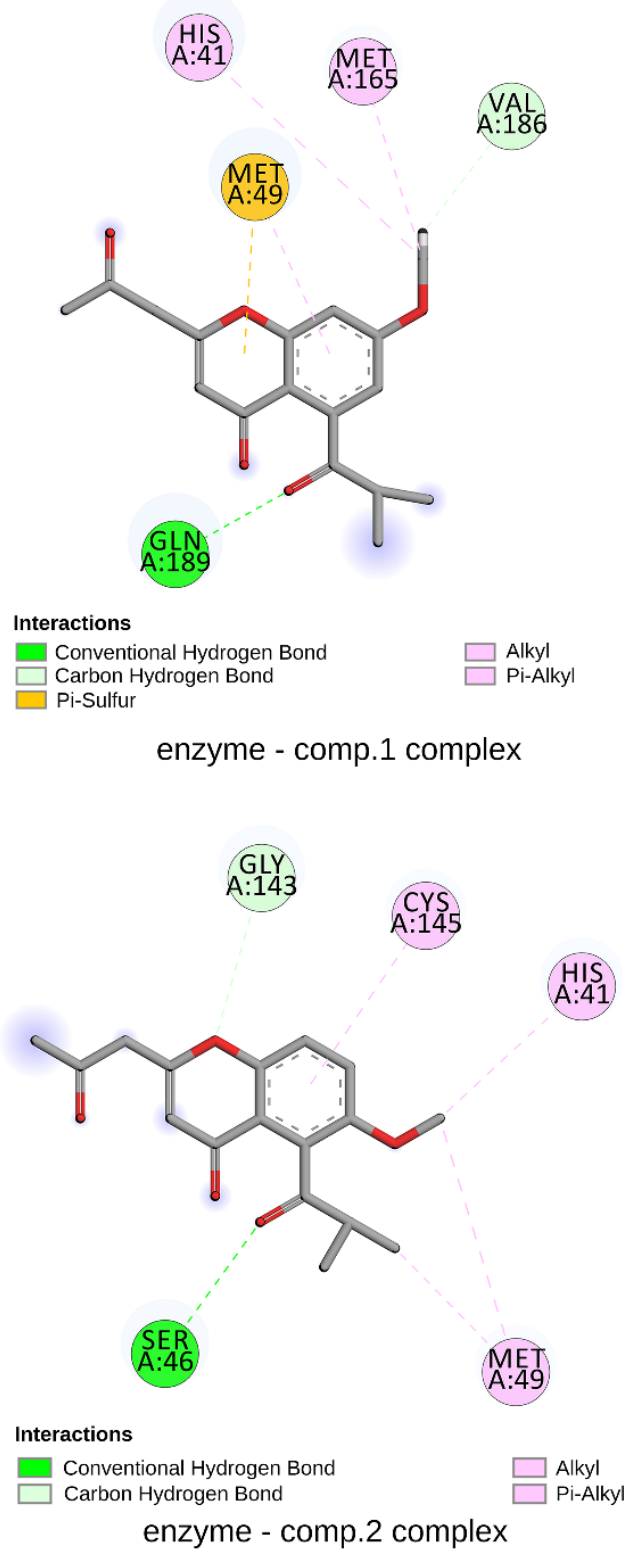

Figure 7. Interactions between ligands and enzyme after MD simulation.

Molecular dynamics simulation results for enzyme Comp. 1 complex are illustrated in Figure 8. Root mean square deviation (RMSD) and radius of gyration (RG) of protein are useful tools for determining the stability of a ligand-protein complex. Results showed that enzyme - Comp. 1 complex remained stable during the MD simulation (Figure $8 \mathrm{~b}$ and $8 \mathrm{c}$ ). Average RMSD of backbone after least square fit to backbone was found to be $0.189 \pm 0.021 \mathrm{~nm}$ and average $\mathrm{RG}$ of protein was found to be $2.224 \pm 0.014 \mathrm{~nm}$. It was observed that there is a noticeable change in the position of Comp. 1 around $7 \mathrm{~ns}$ and then, a slight change around $18 \mathrm{~ns}$. Results showed that ligand reached its equilibrium position at the $18^{\text {th }} \mathrm{ns}$ of the simulation and held its position for the remaining time of the simulation (Figure 8a). Average RMSD of Comp. 1 after least square fit to protein was found to be $0.499 \pm 0.152 \mathrm{~nm}$. Number of hydrogen bonds was also monitored during the MD simulation (Figure 8d). Results showed that at least one hydrogen bond was formed between the ligand and the enzyme in nearly one third of the simulation time. 

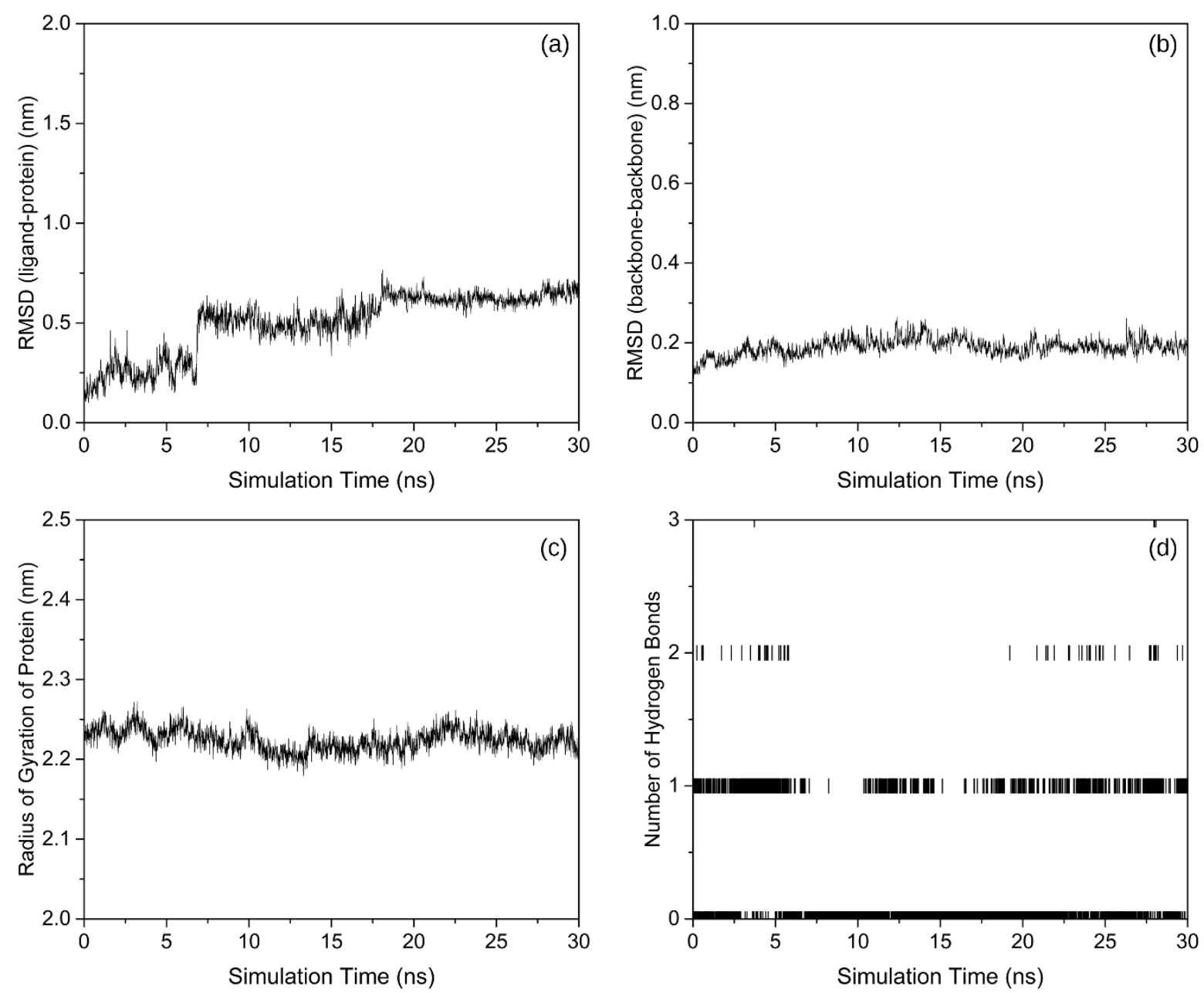

Figure 8. MD simulation results (a) RMSD of ligand after least square fit to protein, (b) RMSD of backbone after least square fit to backbone, (c) Radius of gyration of protein and (d) number of hydrogen bonds between protein and Comp. 1.

Molecular dynamics simulation results for enzyme Comp. 2 complex are illustrated in Figure 9. Results showed that enzyme remained stable during the MD simulation (Figure 9b and 9c). Average RMSD of backbone after least square fit to backbone was found to be $0.219 \pm 0.031 \mathrm{~nm}$ and average $\mathrm{RG}$ of protein was found to be $2.212 \pm 0.010 \mathrm{~nm}$. It was observed that RMSD of ligand after least square fit to protein increased until $17^{\text {th }} \mathrm{ns}$, and then it decreased gradually and reached its equilibrium position at the $24^{\text {th }} \mathrm{ns}$ of the MD simulation (Figure 9a). Average RMSD of Comp. 2 after least square fit to protein was found to be 0.612 $\pm 0.173 \mathrm{~nm}$. Results showed that at least one hydrogen bond was formed between Comp. 2 and the enzyme in nearly half of the simulation time. 

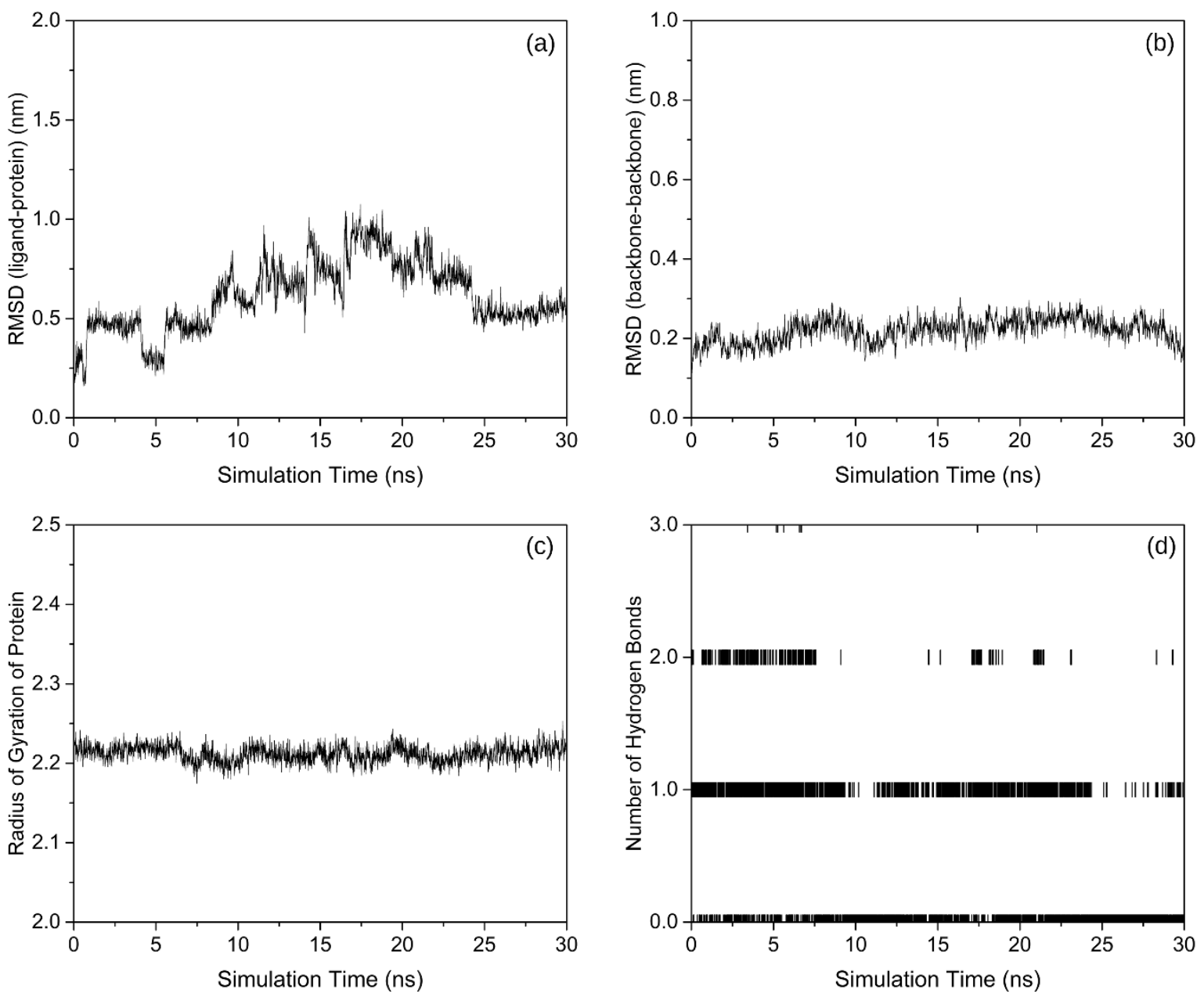

Figure 9. MD simulation results (a) RMSD of ligand after least square fit to protein, (b) RMSD of backbone after least square fit to backbone, (c) Radius of gyration of protein and (d) number of hydrogen bonds between protein and Comp. 2.

Binding free energies were calculated with the use of MM-PBSA method (Figure 10). Binding free energies of the reference drugs are literature values which were determined in our previous work. [8] Results showed that binding affinities of the investigated compounds are quite high. It was observed that although the binding affinity of Comp. 1 is lower than those of lopinavir and hydroxychloroquine, it was higher than that of remdesivir.

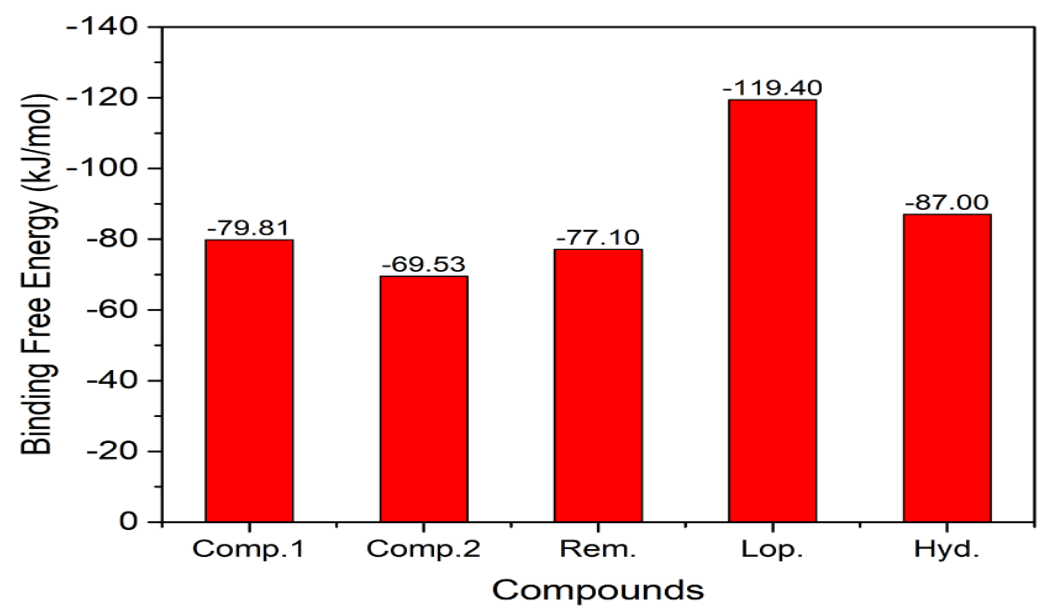

Figure10. Binding free energies belong to investigated compounds and reference drugs obtained from MM-PBSA calculations [8] (Rem.: Remdesivir, Lop.:Lopinavir and Hyd.: Hydroxychloroquine). 


\subsection{Drug-likeness analysis}

Lipinski's rule of five $[25,26]$ was used to evaluate drug-likeness of the investigated compounds. Druglikeness of the investigated compounds were tested with the use of DruLiTo software package [27]. As known, Lipinski's rule describes the molecular properties which are important for a drug's pharmacokinetics, including absorption, distribution, metabolism, and excretion. According to Lipinski's rule a potential drug molecule has no more than one violation of the following criteria:

- A molecular mass less than 500 daltons

- An octanol-water partition coefficient $(\log \mathrm{P})$ that doesn't exceed 5

- No more than 5 HBD (hydrogen bond donors)

- No more than 10 HBA (hydrogen bond acceptors)

For the investigated molecules (both Comp. 1 and 2), molecular weight, $\log \mathrm{P}, \mathrm{HBD}$ and HBA values were found to be 302.12, 0.999, 0 and 5, respectively (Table 6). Results showed that there is no violation of the criteria of Lipinski's rule.

Table 6. Results of drug-likeness analyses.

\begin{tabular}{llll}
\hline \multirow{2}{*}{ Parameter } & \multirow{2}{*}{ Criteria } & \multicolumn{2}{c}{ Calculated Value } \\
& & Comp. 1 & Comp. 2 \\
\hline MW & $<500$ & 302.12 & 302.12 \\
$\log \mathrm{P}$ & $<5$ & 0.999 & 0.999 \\
$\mathrm{HBD}$ & $<5$ & 0 & 0 \\
HBA & $<10$ & 5 & 5 \\
\hline
\end{tabular}

\section{Conclusion}

In the present study, two newly introduced chromone derivatives have been investigated for their possible antiviral activity against SARS-CoV-2 with the assistance of computational methods including DFT calculations, molecular docking calculations and molecular dynamics simulations. In the study, MMPBSA calculations and drug-likeness analyses were also carried out. Results showed that investigated compounds efficiently bound to SARS-CoV-2 $\mathrm{M}^{\text {pro }}$ and held their positions throughout the entire molecular dynamics simulations. Binding affinities of the investigated compounds were found to be quite high and comparable to the reference drugs. Especially binding affinity of Comp. 1 is higher than that of Comp. 2 and reference drug remdesivir. However, it was found that the binding affinities of the investigated compounds are lower than those of lopinavir and hydroxychloroquine. Additionally, it was observed that both compounds obey Lipinski's rule of five and no violation was observed. In the study it was concluded that these natural chromone derivatives can be promising structures in the treatment of SARSCoV-2 infections and worth for further research.

\section{Acknowledgements}

The computational studies reported in this paper were partially performed at TUBITAK ULAKBIM, High Performance and Grid Computing Center (TRUBA resources) and Kocaeli University.

\section{Conflict of interest}

The authors declare that they have no conflict of interests.

\section{References}

[1] Cheng C.Y., Lee Y.L., Chen C.P., Lin Y.C., Liu C.E., Liao C.H., Cheng S.H., Lopinavir/ritonavir did not shorten the duration of SARS CoV-2 shedding in patients with mild pneumonia in Taiwan, Journal of Microbiology, Immunology and Infection, 53 (2020) 488-492.

[2] Lin M.H., Moses D.C., Hsieh C.H., Cheng S.C., Chen Y.H., Sun C.Y., Chou C.Y., Disulfiram can inhibit MERS and SARS coronavirus papain-like proteases via different modes, Antiviral Research, 150 (2018) 155-163.

[3] Yu R., Chen L., Lan R., Shen R., Li P., Computational screening of antagonists against the SARS-CoV-2 (COVID-19) coronavirus by molecular docking, International Journal of Antimicrobial Agents, 56(2) (2020) 106012.

[4] Elfiky A.A., Ribavirin, Remdesivir, Sofosbuvir, Galidesivir and Tenofovir against SARS-CoV-2 RNA dependent RNA polymerase (RdRp): A molecular docking study, Life Sciences, 253 (2020).

[5] Cai Q., Yang M., Liu D., Chen J., Shu D., Xia J., Liao X., Gu Y., Cai Q., Yang Y., Shen C., Li X., Peng L., Huang D., Zhang J., Zhang S., Wang F., Liu J., Chen L., Chen S., Wang Z., Zhang Z., Cao R., Zhong W., Liu Y., Liu L., Experimental Treatment with Favipiravir for COVID-19: An Open-Label Control Study, Engineering, 6 (2020) 1192-1198. 
[6] Choy K.T.,. Wong A.Y.L, Kaewpreedee P., Sia S.F., Chen D., Hui K.P.Y., Chu D.K.W., Chan M.C.W., Cheung P.P.H., Huang X., Peiris M., Yen H.L., Remdesivir, lopinavir, emetine, and homoharringtonine inhibit SARS-CoV-2 replication in vitro, Antiviral Research, 178 (2020) 104786.

[7] McKee D.L., Sternberg A., Stange U., Laufer S., Naujokat C., Candidate drugs against SARSCoV-2 and COVID-19, Pharmacological Research, 157 (2020) 104859.

[8] Erdogan T., DFT, molecular docking and molecular dynamics simulation studies on some newly introduced natural products for their potential use against SARS-CoV-2, Journal of Molecular Structure, 1242 (2021) 130733.

[9] Liu X., Wang X.J., Potential inhibitors against 2019-nCoV coronavirus $M$ protease from clinically approved medicines, Journal of Genetics and Genomics, 47 (2020) 119-121.

[10] Xu Z., Peng C., Shi Y., Zhu Z., Mu K., Wang X., Zhu W., Nelfinavir was predicted to be a potential inhibitor of 2019-nCov main protease by an integrative approach combining homology modelling, molecular docking and binding free energy calculation, BioRxiv, (2020) 2020.01.27.921627.

[11] Liao L.M., Sun Y.Q., Li J., Kong W.S., Liu X., Xu Y., Huang H.T., Zeng W.L., Mi Q.L., Yang G.Y., Hu Q.F., Li Y.K., Two New Chromone Derivatives from Cassia nomame and their AntiTobacco Mosaic Virus Activity, Chemistry of Natural Compounds, 56 (2020) 58-61.

[12] Frisch M.J., Trucks G.W., Schlegel H.B., Scuseria G.E., Robb M.A., Cheeseman J.R., Scalmani G., Barone V., Mennucci B., Petersson G.A., Nakatsuji H., Caricato M., Li X., Hratchian H.P., Izmaylov A.F., Bloino J., Zheng G., Sonnenberg J.L., Hada M., Ehara M., Toyota K., Fukuda R., Hasegawa J., Ishida M., Nakajima T., Honda Y., Kitao O., Nakai H., Vreven T., Montgomery J. A., Peralta J.E., Ogliaro F., Bearpark M., Heyd J.J., Brothers E., Kudin K.N., Staroverov V.N., Keith T., Kobayashi R., Normand J., Raghavachari K., Rendell A., Burant J.C., Iyengar S.S., Tomasi J., Cossi M., Rega N., Millam J.M., Klene M., Knox J.E., Cross J.B., Bakken V., Adamo C., Jaramillo J., Gomperts R., Stratmann R.E., Yazyev O., Austin A.J., Cammi R., Pomelli C., Ochterski J.W., Martin R.L., Morokuma K., Zakrzewski V.G., Voth G.A., Salvador P., Dannenberg J.J., Dapprich S., Daniels A.D.,
Farkas O., Foresman J.B., Ortiz J. V, Cioslowski J., Fox D.J., Gaussian 09, (2013).

[13] Dennington, R., T. Keith, J. Millam, GaussView, Version 5, (2009).

[14] Chang C. E. Gilson M.K., Tork: Conformational analysis method for molecules and complexes, Journal of Computational Chemistry, 24 (2003) 1987-1998.

[15] Hanwell M.D., Curtis D.E., Lonie D.C., Vandermeersch T., Zurek E., Hutchison G.R., Avogadro: an advanced semantic chemical editor, visualization, and analysis platform, Journal of Cheminformatics, 4 (2012) 17.

[16] Morris G.M., Huey R., Lindstrom W., Sanner M.F., Belew R.K., Goodsell D.S., Olson A.J., AutoDock4 and AutoDockTools4: Automated docking with selective receptor flexibility, $J$ Comput. Chem., 30 (2009) 2785-2791.

[17] BIOVIA, D.S., Discovery Studio Visualizer, v20.1.0.19295, (2016).

[18] RCSB PDB, Available at: https://www.rcsb.org/, Retrieved May, 2021.

[19] Berman H.M., Westbrook J., Feng Z., Gilliland G., Bhat T.N., Weissig H., Shindyalov I.N., Bourne P.E., The Protein Data Bank, Nucleic Acids Research, 28 (2000) 235-242.

[20] Lindahl, Abraham, Hess, van der Spoel, GROMACS 2020 Source code, (2020).

[21] Ponder J.W., Case D.A., Force fields for protein simulations, Advances in Protein Chemistry, 66 (2003) 27-85.

[22] Sousa Da Silva A.W., Vranken W.F., ACPYPE AnteChamber PYthon Parser interfacE, $B M C$ Research Notes, 5 (2012) 367.

[23] Kumari R., Kumar R., Lynn A., G-mmpbsa -A GROMACS tool for high-throughput MM-PBSA calculations, Journal of Chemical Information and Modeling, 54 (2014) 1951-1962.

[24] Baker N.A., Sept D., Joseph S., Holst M.J., McCammon J.A., Electrostatics of nanosystems: Application to microtubules and the ribosome, Proceedings of the National Academy of Sciences of the United States of America, 98 (2001) 10037 10041.

[25] Lipinski C.A., Lombardo F., Dominy B.W., Feeney P.J., Experimental and computational approaches to estimate solubility and permeability in drug discovery and development settings, Advanced Drug Delivery Reviews, 46 (2001) 3- 
26.

[26] Lipinski C.A., Lombardo F., Dominy B.W., Feeney P.J., Experimental and computational approaches to estimate solubility and permeability in drug discovery and development settings, Advanced Drug Delivery Reviews, 23 (1997) 325.

[27] Drug Likeness Tool (DruLiTo), Available at: http://www.niper.gov.in/pi_dev_tools/DruLiTo W eb/DruLiTo_index.html, Retrieved May, 2020. 